\title{
Réactions d'immunité cellulaire après implantation de cellophane et dans la tumorigenèse chez les Insectes
}

\author{
par G. MATZ \\ Laboratoire de Biologie animale, Faculté des Sciences d'Angers, \\ boulevard Lavoisier, F 49045 Angers Cedex
}

Les réactions d'immunité cellulaire ont été étudiées chez l'Orthoptère Locusta migratoria et différentes espèces de Dictyoptères après implantation d'un corps étranger inerte et dans la tumorigenèse.

La réaction immunitaire des insectes envers un parasite est relativement bien connue car elle a fait l'objet d'études et de travaux expérimentaux. Afin de simplifier l'interprétation et d'en exclure le facteur \&vivant ", nous avons implanté dans la cavité hémolymphatique un fragment de cellophane, corps étranger inerte. Celui-ci, dès la deuxième heure de son séjour dans l'abdomen de l'insecte, se trouve entouré d'une couche de cellules sanguines qui continuent à affluer pour former une capsule hémocytaire. La capsule résulte de l'accumulation, sans division mitotique, d'hémocytes principalement de type granuleux. Elle est formée d'un nombre variable d'assises cellulaires; ce nombre peut approcher 20 , mais ne dépend pas de la durée de l'expérience. Dans le cytoplasme des cellules étirées parallèlement au corps étranger apparaissent des microfilaments et des microtubules : c'est la «capsule conjonctive stabilisée » (Matz, 1965) dans laquelle le corps étranger se trouve isolé. La quasi-totalité des tumeurs spontanées, décrites chez les insectes, ainsi que les formations spontanées observées par nous chez les espèces utilisées pour nos expériences ne représentent que des accumulations d'hémocytes consécutives à des blessures, à des dégénérescences tissulaires ou à la présence de parasites comme les grégarines. Les structures d'encapsulement se différencient des tumeurs épithéliales que nous allons évoquer plus loin par l'absence de prolifération cellulaire, l'absence de métastases et l'impossibilité de transmission par un filtrat.

La réaction hémocytaire d'immunité cellulaire, qui existe toujours seule chez Blabera craniifer, peut être renforcée par la mise en place d'un hypoderme au sein 
de la capsule hémocytaire : un épithélium d'origine trachéenne ou parfois tégumentaire apparaît selon les conditions expérimentales ou selon l'espèce. Nous l'avons observé rarement ( $6 \%$ des animaux en expérience) chez Periplaneta americana, plus fréquemment $(21 \%)$ chez Leucophaea maderae et chez la totalité des animaux opérés chez le Coléoptère Pimelia cribripennis. Enfin, chez Locusta migratoria, cet hypoderme n'apparaît que dans la capsule entourant un implant non stérile.

La section du nerf récurrent provoque, chez Locusta migratoria et Leucophaea maderae, l'apparition de tumeurs. Celles-ci se développent par multiplication cellulaire de l'épithélium de l'organe atteint. Cette prolifération épithéliale est surtout très nette au niveau des organes endodermiques: dans les nids de régénération, l'activité mitotique s'accroît significativement; le pourcentage des cellules en division chez les animaux à nerf récurrent sectionné est sensiblement égal au double de celui des animaux témoins. Puis on retrouve le mécanisme de défense cellulaire hémocytaire car les hémocytes viennent s'insinuer entre les cellules épithéliales tumorales qui se trouvent encapsulées. Les tumeurs, transmissibles par filtrat acellulaires, se trouvent alors formées d'un mélange des deux types cellulaires qui peuvent coexister, les cellules épithéliales poursuivant leur multiplication.

En résumé, l'encapsulement d'un corps étranger inerte se réalise par accumulation d'hémocytes, sans division mitotique; les cellules épithéliales tumorales, formant à la suite de divisions cellulaires des tumeurs épithéliales, sont également encapsulées par les hémocytes.

\section{Bibliographie}

Boufersaoui (A.) et Matz (G.), 1974. - Augmentation de l'activité mitotique dans l'intestin moyen après section du nerf récurrent chez l'imago de Leucophaea maderae Fabr. (Insecte, Dictyoptère). C.R. Acad. Sci., D, 279, 1889-1890.

Brehelin (M.), Hoffmann (J.-A.), Matz (G.) et Porte (A.), 1975. - Encapsulation of implanted foreign bodies by hemocytes in Locusta migratoria and Melolontha melolontha. Cell Tiss. Res., 160, 283-289.

Matz (G.), 1965. - Implantation de fragments de cellophane chez Locusta migratoria L. (Orthoptère). Bull. Soc. zool. Fr., 90, 429-433.

Matz (G.), 1967. - Etude du cancer expérimental chez Locusta migratoria L. (Insecte, Orthoptère). Biol. Méd., 56, 454-490.

Matz (G.), 1969. - Manifestations inflammatoires et tumorales chez les Orthoptères. Ann. Zool. Ecol. animale, 1 ( $\mathrm{n}^{\circ}$ hors-ś́rie), 49-62.

Matz (G.), Monier Y.) et Vago (C.), 1971. - Une réaction de défense cellulaire chez les Insectes: l'enkystement épithélial. Bull. Soc. zool. Fr., 96, 209-215. 